\title{
Tau Isoforms Imbalance Impairs the Axonal Transport of the Amyloid Precursor Protein in Human Neurons
}

\author{
Valentina Lacovich, ${ }^{1,2 *}$ Sonia L. Espindola, ${ }^{3 \star}$ Matías Alloatti, ${ }^{1 *}$ Victorio Pozo Devoto, ${ }^{1}$ Lucas E. Cromberg, ${ }^{1}$ \\ Mária E. Čarná, ${ }^{2}$ Giancarlo Forte, ${ }^{2}$ Jean-Marc Gallo, ${ }^{4}$ Luciana Bruno, ${ }^{5}$ Gorazd B. Stokin, ${ }^{2}$ M. Elena Avale, ${ }^{3}$ \\ and Tomás L. Falzone ${ }^{1,6}$ \\ ${ }^{1}$ Instituto de Biología Celular y Neurociencias (IBCN-CONICET-UBA), Facultad de Medicina, Universidad de Buenos Aires, Buenos Aires CP 1121, \\ Argentina, ${ }^{2}$ Centre for Translational Medicine (CTM), International Clinical Research Center, St. Anne's University Hospital (ICRC-FNUSA), Brno 65691, \\ Czech Republic, ${ }^{3}$ Instituto de Investigaciones en Ingeniería Genética y Biología Molecular (INGEBI-CONICET), Buenos Aires C1428ADN, Argentina, \\ ${ }^{4}$ Maurice Wohl Clinical Neuroscience Institute, Institute of Psychiatry, Psychology and Neuroscience, King's College London, London SE5 9NU, United \\ Kingdom, ${ }^{5}$ Departamento de Física (IFIBA-CONICET), Facultad de Ciencias Exactas y Naturales (UBA), Buenos Aires C1428EGA, Argentina, and ${ }^{6}$ Instituto \\ de Biología y Medicina Experimental (IBYME-CONICET), Buenos Aires C1428ADN, Argentina
}

Tau, as a microtubule (MT)-associated protein, participates in key neuronal functions such as the regulation of MT dynamics, axonal transport, and neurite outgrowth. Alternative splicing of exon 10 in the tau primary transcript gives rise to protein isoforms with three (3R) or four (4R) MT binding repeats. Although tau isoforms are balanced in the normal adult human brain, imbalances in 3R:4R ratio have been tightly associated with the pathogenesis of several neurodegenerative disorders, yet the underlying molecular mechanisms remain elusive. Several studies exploiting tau overexpression and/or mutations suggested that perturbations in tau metabolism impair axonal transport. Nevertheless, no physiological model has yet demonstrated the consequences of altering the endogenous relative content of tau isoforms over axonal transport regulation. Here, we addressed this issue using a trans-splicing strategy that allows modulating tau exon 10 inclusion/exclusion in differentiated human-derived neurons. Upon changes in 3R:4R tau relative content, neurons showed no morphological changes, but live imaging studies revealed that the dynamics of the amyloid precursor protein (APP) were significantly impaired. Single trajectory analyses of the moving vesicles showed that predominance of $3 \mathrm{R}$ tau favored the anterograde movement of APP vesicles, increasing anterograde run lengths and reducing retrograde runs and segmental velocities. Conversely, the imbalance toward the $4 \mathrm{R}$ isoform promoted a retrograde bias by a significant reduction of anterograde velocities. These findings suggest that changes in 3R:4R tau ratio has an impact on the regulation of axonal transport and specifically in APP dynamics, which might link tau isoform imbalances with APP abnormal metabolism in neurodegenerative processes.

Key words: Alzheimer's; APP; axonal transport; splicing; tau; tauopathies

Significance Statement

The tau protein has a relevant role in the transport of cargos throughout neurons. Dysfunction in tau metabolism underlies several neurological disorders leading to dementia. In the adult human brain, two tau isoforms are found in equal amounts, whereas changes in such equilibrium have been associated with neurodegenerative diseases. We investigated the role of tau in human neurons in culture and found that perturbations in the endogenous balance of tau isoforms were sufficient to impair the transport of the Alzheimer's disease-related amyloid precursor protein (APP), although neuronal morphology was normal. Our results provide evidence of a direct relationship between tau isoform imbalance and defects in axonal transport, which induce an abnormal APP metabolism with important implications in neurodegeneration.

\section{Introduction}

The microtubule (MT)-associated protein tau regulates MT dynamics, supporting the axonal transport of proteins, vesicles, and

Received July 20, 2016; revised Sept. 21, 2016; accepted Nov. 4, 2016

Author contributions: J.-M.G., L.B., G.B.S., M.E.A., and T.L.F. designed research; V.L., S.L.E., M.A., V.P.D., L.E.C., M.E.A., and T.L.F. performed research; G.F. contributed unpublished reagents/analytic tools; S.L.E., M.A., M.C., L.B., M.E.A., and T.L.F. analyzed data; M.E.A. and T.L.F. wrote the paper. organelles (for review, see Morris et al., 2011). A number of neurodegenerative diseases referred to as tauopathies, including Alzheimer's disease and some forms of frontotemporal dementia, 
are characterized by the aberrant accumulation of hyperphosphorylated tau. In such pathological conditions, tau is disengaged from MTs and accumulates in insoluble filamentous inclusions (for review, see Spillantini and Goedert, 2013).

The human MAPT gene encoding tau protein comprises 16 exons. Alternative splicing of exons 2, 3, and 10 produces six different isoforms in the CNS (Goedert et al., 1989). Exon 10 (E10) encodes the second of four MT-binding repeats; therefore, E10 alternative splicing gives rise to tau isoforms with three (3R) or four (4R) MT-binding repeats (Goedert et al., 1989). Only 3R tau is expressed in the developing brain; a balanced 3R:4R tau expression is found in the adult human brain (Goedert et al., 1989; Andreadis, 2005). Mutations impairing E10 splicing segregate with disease in familial frontotemporal dementia with parkinsonism linked to chromosome 17 (FTDP-17) (Hutton et al., 1998; Spillantini et al., 1998). To date, $>50$ disease-associated mutations have been found in MAPT, with one-third of them affecting E10 splicing (Ghetti et al., 2015). However, the mechanisms underlying neurodegeneration due to $3 \mathrm{R}: 4 \mathrm{R}$ isoform imbalance remain to be further elucidated.

Among the myriad of neuronal functions affected by tau abnormal metabolism, axonal transport is likely to be particularly disturbed: The anterograde and retrograde axonal transport of cargos is mediated by kinesin and dynein motors that interact differentially within the MT polarity (Terada et al., 2010; Encalada and Goldstein, 2014). Tau can regulate axonal transport by interacting directly with molecular motors, altering MT dynamics, or competing with the binding of motors to MT (Dixit et al., 2008; Goldstein, 2012; Kanaan et al., 2012). The tau-MT interaction depends on the number of tau MT-binding domains (Panda et al., 2003), so it is potentially affected by the relative content of $3 \mathrm{R}$ and $4 \mathrm{R}$ isoforms, as well as by mutations in E10. Transgenic mice expressing missense human E10 mutations (P301S or P301L) revealed deficits in axonal transport (Ittner et al., 2008; Bull et al., 2012; Mellone et al., 2013; Rodríguez-Martín et al., 2016). Conversely, reduction in the level of kinesin-I anterograde motor enhances tau pathology in the P301L transgenic mouse, suggesting that transport defects affect tau physiology (Falzone et al., 2009). In addition, cultured neurons showed mitochondrial axonal transport defects under 3R or 4R isoform overexpression (Mertens et al., 2013) or when bearing mutations that increase E10 inclusion (Iovino et al., 2014).

The amyloid precursor protein (APP) is significantly linked to Alzheimer's disease, leading to the generation of amyloid- $\beta$ and plaque pathology under the presence of mutations, overexpression, or transport defects (Stokin et al., 2005; Goldstein, 2012). As a Golgi-derived transmembrane protein, APP vesicles undergo constitutive fast axonal transport, interacting with kinesin and dynein motors (Reis et al., 2012, Fu and Holzbaur, 2013, Koo et al., 1990). Defects in APP transport are early events in neurode-

1.1.00/02.0123 and LQ1605 to G.B.S.). T.L.F and M.E.A were supported by Consejo Nacional de Investigaciones Cientificas y Técnicas, University of Buenos Aires, and the Argentinean Science Ministry. S.L.E., V.P.D., M.A., and L.E.C. receive fellowships from CONICET. We thank Drs. Mariela Sued and Daniela Rodríguez for help with the statistical analysis of the data; Roux-Ocefa Argentina for kindly contributing basic reagents, and Fundación Rene Baron for donations.

The authors declare no competing financial interests.

*V.L., S.L.E., and M.A. contributed equally to this work.

Correspondence should be addressed to either of the following: Tomás L. Falzone, Instituto de Biología Celulary Neurociencias (IBCN-CONICET-UBA), Paraguay 2155, Facultad de Medicina, Universidad de Buenos Aires, Buenos Aires CP 1121, Argentina, E-mail: tfalzone@fmed.uba.ar; or M. Elena Avale, Instituto de Investigaciones en Ingeniería Genética y Biología Molecular (INGEBI-CONICET), Vuelta de Obligado 2490, Buenos Aires C1428ADN, Argentina. E-mail: elena.avale@conicet.gov.ar.

DOI:10.1523/JNEUROSCI.2305-16.2016

Copyright $\odot 2017$ the authors $\quad 0270-6474 / 17 / 370059-12 \$ 15.00 / 0$ generative diseases (Stokin et al., 2005; De Vos et al., 2008); therefore, measuring APP dynamics is the gold standard method for detecting transport deficits associated with pathological conditions (Goldsbury et al., 2007; Falzone and Stokin, 2012).

In this study, we investigated whether the imbalance in $3 \mathrm{R}: 4 \mathrm{R}$ ratio affect the dynamics of APP axonal transport in human neurons. We used a trans-splicing RNA reprogramming strategy that drives the inclusion/exclusion of exon 10 in the endogenous tau transcript (Avale et al., 2013). The tau isoform ratio was modulated without altering total tau protein amount in cultured differentiated neurons derived from human embryonic stem cells (hESCs). Live-imaging analyses of APP fluorescent vesicles revealed differential effects of $3 \mathrm{R}$ or $4 \mathrm{R}$ isoforms over anterograde and retrograde axonal transport, suggesting that changes in endogenous tau isoform ratio impairs APP transport in human neurons.

\section{Materials and Methods}

Cell culture and neuronal differentiation. Neurons were derived from hESCs. Briefly, irradiated murine embryonic fibroblasts were plated over gelatin-coated Petri dishes $24 \mathrm{~h}$ before hESC plating and maintained in DMEM complete (high-glucose DMEM, 10\% FBS, 1\% Glutamax, 1\% penicillin-streptomycin). hESCs were grown at $37^{\circ} \mathrm{C}, 95 \%$ humidity, and $5 \% \mathrm{CO}_{2}$ in hESC medium (KO DMEM, 20\% KO serum replacement, $1 \%$ Glutamax, $1 \%$ nonessential amino acids, $0.1 \%$ betamercaptoethanol, $4 \mathrm{ng} / \mathrm{ml} \mathrm{bFGF}$ ) to allow colony formation. When colonies reached optimal size, they were first transferred to a Petri dish and, after $12-18 \mathrm{~h}$, to $25 \mathrm{~cm}^{2}$ flasks and grown in suspension to allow embryoid body formation, which was induced by neural induction medium (NIM; DMEM/F12, 1\% N2 supplement, 1\% nonessential amino acids, $280 \mathrm{UI} / \mathrm{ml}$ heparin, and $1 \%$ penicillin-streptomycin). Embryoid bodies were then transferred to laminin-coated six-well plates for neural rosette formation. After 7-14 d of growth, the neural rosettes were picked and transferred to $25 \mathrm{~cm}^{2}$ flasks and maintained in NIM complete medium (2\% B27 supplement, ascorbic acid) for up to 1 month, changing the growth medium every $2 \mathrm{~d}$. Neural rosettes were picked and dissociated by a $5 \mathrm{~min}$ of accutase and trypsin treatment. The reaction was blocked by trypsin inhibitor and the suspension centrifuged for $5 \mathrm{~min}$ at $1000 \mathrm{rpm}$. The pellet was washed with DMEM/F12 and resuspended in neural differentiation media (NDM; Neurobasal, 1\% N2 supplement, 2\% B27 supplement, and 1\% penicillin-streptomycin). Cells were plated over poly-ornitine and laminin-coated coverslips into 24-well plates and maintained in $500 \mu \mathrm{l} /$ well NDM complete medium (laminin, cAMP, ascorbic acid, BDNF, GDNF).

Neuron plating, transduction, and transfection. Plated neurons were maintained in culture, changing $3 / 4$ of the medium every $7 \mathrm{~d}$. On $\mathrm{d}$ in vitro 11 (DIV11), neurons were transduced with control (dsRED), PTM-4R (formerly named PTM6) (Rodriguez-Martin et al., 2005), or PTM-3R (formerly PTM9) (Rodriguez-Martin et al., 2009). PTMs were expressed under the human synapsin promoter in a replication-defective lentiviral vector (LV) prepared as described previously (Avale et al., 2013). Then, $5 \mu \mathrm{l}$ of each concentrated $\mathrm{LV}\left(\sim 10^{7} \mathrm{TU} / \mathrm{ml}\right)$ was added in $200 \mu \mathrm{l}$ of medium per well containing 150,000 neurons to achieve a multiplicity of infection between 5 and 10 , as described previously (Avale et al., 2013). After $12 \mathrm{~h}$, the cells were topped up with $300 \mu \mathrm{l}$ of fresh NDMc medium. Seven days after LV transduction (on DIV18), neurons were transfected with $1 \mu \mathrm{g}$ of pcDNA3-APP-YFP (Falzone et al., 2009) in a transfection mixture of OptiMEM and Lipofectamine 2000. Two hours after transfection, the culture medium was replaced and, $24 \mathrm{~h}$ later (on DIV19), neurons were analyzed by imaging for transport analysis, fixed for immunocytochemistry, or processed to obtain RNA and protein for qPCR and Western blot analyses.

$3 R$ and $4 R$ tau isoform quantitation by $q P C R$. Total RNA was isolated from neurons using the AllPrep DNA/RNA mini kit (Qiagen) from highdensity cultures at DIV19. Reverse transcription was performed with $0.5 \mu \mathrm{g}$ of RNA with the TaqMan RT kit (Applied Biosystems) in a total volume of 10 
$\mu$ l. RT conditions were as follows: $10 \mathrm{~min}$ at $25^{\circ} \mathrm{C}, 30 \mathrm{~min}$ at $48^{\circ} \mathrm{C}$, and $5 \mathrm{~min}$ at $95^{\circ} \mathrm{C}$. To simultaneously detect tau isoforms by end-point PCR, $0.2 \mu \mathrm{g}$ of reverse-transcribed RNA was amplified by PCR using Go Taq polymerase (Promega) with primers spanning exons $9-13$, as described previously (Avale et al., 2013). PCR products were separated by electrophoresis in $2 \%$ $(\mathrm{w} / \mathrm{v})$ agarose gels and stained with ethidium bromide. To perform the relative quantification of $4 \mathrm{R}$ and $3 \mathrm{R}$ mRNA isoforms by real-time PCR, specific pairs of primers were used for each isoform, annealing with the E9-E10 or the E9/11-E11 junction. Both pairs of primers showed similar efficiency. Primer sequences were as follows: 4Rfor(E9): $5^{\prime}$-TCCACTGAGAACCTGAAG-3'; 4Rrev(E10): 5' -AGTGTGGCTCAAAGGATA-3'; 3Rfor(E9/11): 5'-AGGCGGGAAGGTGCAAATAG-3'; 3Rrev(E11): 5' -TCCTGGTTTATGATGGATGTT- $3^{\prime}$. The mRNA coding for human apolipoprotein B (ApoB) was used as a reference (ApoBFor: $5^{\prime}$-TGGTGCTCACAAGGCGACACTAA-3'; ApoBRev: 5'-GGCGGCTTTCCCATCCAGACTA-3'). Realtime PCRs were performed in triplicate with $10 \mathrm{ng}$ of cDNA and $5 \mu \mathrm{l}$ of Power SYBR Green PCR Master Mix (Applied Biosystem) in a final volume of $10 \mu \mathrm{l}$ using an MJ Research Opticon 2 thermal cycler under the following cycling conditions: initial denaturation at $95^{\circ} \mathrm{C}$ for $3 \mathrm{~min}, 50$ cycles of $30 \mathrm{~s}$ at $95^{\circ} \mathrm{C}, 30 \mathrm{~s}$ at $58^{\circ} \mathrm{C}$, and $45 \mathrm{~s}$ at $72^{\circ} \mathrm{C}$ and a final step of $1 \mathrm{~min}$ at $72^{\circ} \mathrm{C}$. Data were analyzed with Opticon Monitor 3 software (Bio-Rad) to obtain the $\Delta \mathrm{Ct}$ per sample (Ct tau isoforms - Ct ApoB). Data from three independent experiments were pooled and analyzed using GraphPad Prism software.

Western blotting. Total protein was collected from neurons at DIV19 in $100 \mu$ l of sample buffer (40 mu Tris- $\mathrm{HCl}, \mathrm{pH} 7.5,150 \mathrm{~mm} \mathrm{NaCl}$ in $1 \times$ protease inhibitor; Invitrogen). Protein concentration was measured using the Bradford assay (Bio-Rad). Equal amounts of protein $(10-20 \mu \mathrm{g})$ in $20 \mu \mathrm{l}$ of loading buffer $(0.5 \%$ bromophemol blue, $10 \%$ glycerol, and $10 \% 2$-mercaptoethanol) were loaded onto $12 \%(\mathrm{w} / \mathrm{v})$ SDSpolyacrylamide gels $\left(N, N^{\prime}\right.$-methylenebisacrilamide $30 \%$; Invitrogen). Proteins were transferred onto nitrocellulose membranes (Whatman) using the wet transfer method. Membranes were blocked in 5\% (w/v) nonfat milk (Sancor) and $0.05 \% \mathrm{v} / \mathrm{v}$ Tween 20 in TBS for $1 \mathrm{~h}$. After blocking, membranes were incubated overnight at $4^{\circ} \mathrm{C}$ in blocking solution containing primary antibody directed against $3 \mathrm{R}$ tau (1:2000 antitau 3-repeat isoform RD3; mouse monoclonal; Millipore), 4R tau (1: 1000 anti-tau 4-repeat isoform RD4; mouse monoclonal; Millipore), total tau (1:10000; rabbit polyclonal; Sigma-Aldrich), phosphorylated tau CP13 and PHF1 (1:200; gift from Dr. Peter Davies), $\beta$-actin (mouse monoclonal, 1:10000; Abcam), APP (mouse monoclonal 22C11), or tubulin (mouse monoclonal, 1:800, DM1a; Covance). After washing 3 times in TBS containing $0.05 \% \mathrm{v} / \mathrm{v}$ Tween 20 , blots were incubated with the appropriate secondary antibody, goat anti-rabbit (1:2000; Cell Signaling Technology) or horse anti-mouse (1:2000; Cell Signaling Technology) for $3 \mathrm{~h}$ at room temperature. Proteins were visualized using the GeneGnomeXRQ (Syngene). Scanned images were analyzed using ImageJ software.

Imaging, tracking, and analysis. Imaging of live cells and kymograph analysis of axonal transport was performed as described previously (Falzone and Stokin, 2012). Briefly, 30 s movies of APP-YFP moving particles in neurons were recorded using an inverted epifluorescence microscope (Olympus IX81) connected to a CCD camera (Olympus DP71/12.5 megapixels). Cultures were observed under a $60 \times$ lens (numerical aperture, NA: 1.40 ) and maintained at $37^{\circ} \mathrm{C}, 5 \% \mathrm{CO}_{2}$, and $10 \%$ humidity using a $\mathrm{CO}_{2}$ humid chamber and heated stage (Olympus). Directionality was determined by tracking fluorescent axons. To avoid introducing biases due to the gradient concentration of tau in axons, imaging was performed in axons at their middle part separated by at least two fields of view distance $(\sim 200 \mu \mathrm{m})$ from cell bodies and from axonal tips. Kymographs were generated from the recordings with ImageJ using the multiple kymograph plug-in (Otero et al., 2014) and processed using Image-Pro Plus 6 (Media Cybernetics) and MATLAB scripts (The MathWorks) to track single fluorescent vesicles with subpixel precision, as described previously (Otero et al., 2014). Kymographs were blind coded for particle tracking and unbiased data collection.

Segmental velocities, run lengths, pauses, and reversions were computed from trajectories using custom-made MATLAB routines. For the calculation of segmental velocities, processive trajectories were divided into segments and the mean velocity of the segment was computed from the slope using a MATLAB algorithm. Briefly, each trajectory was cut in segments of 20 frames of duration, producing a linear approximation with the least-squares method, filtering trajectories with outliers and significant differences between the dataset and the fitted curve. Subsequently, the slope of the regression was considered as the velocity of the segment. The point in which the movement is equal to 0 , filtering the points produced by the noise of the trajectory was used for the calculation of run lengths, pauses, and reversions. The trajectories between those points are considered continuous segments and classified in anterograde or retrograde movement if they show positive or negative velocity respectively (velocities are the slope of a linear approximation of the trajectory). Pauses are considered when segments follow a stationary criteria for $>5$ frames $(625 \mathrm{~ms})$ moving $<0.05$ pixel per frame $(<0.16 \mu \mathrm{m} / \mathrm{s})$. The script merges continuous segments of the same type and segments that are too small. The difference in micrometers between the final point and the initial point of a determined continuous segment is considered as the run length. The points separating anterograde and retrograde segments are considered reversions. The parameters of the Gaussian mixture model were determined by an expectation maximization algorithm using a function (gmdistribution.fit) in MATLAB. A bootstrapping with resampling procedure with $N=1000$ was implemented to compute the intervals of confidence of the Gaussian mixture parameter estimates.

Immunofluorescence, image collection, and morphology. Cells were washed with PBS and fixed with 4\% paraformaldehyde and $4 \%$ sucrose in PBS for 30 min at $37^{\circ} \mathrm{C}$. After fixation, cells were washed twice with PBS for $10 \mathrm{~min}$ and permeabilized with $0.1 \%$ Triton X-100 for $10 \mathrm{~min}$ at room temperature. Cells were incubated at room temperature for $1 \mathrm{~h}$ using a blocking solution consisting of 3\% BSA, $0.1 \%$ Triton X-100, and 10\% goat serum in PBS. Cells were then stained with primary antibodies in blocking solution and incubated overnight at $4^{\circ} \mathrm{C}$. When using two primary antibodies, the staining was done sequentially. Cells were then rinsed in PBS and stained with secondary antibodies at room temperature for $2 \mathrm{~h}$. Cells were stained for $30 \mathrm{~min}$ with Hoechst and mounted on slides with $70 \%$ glycerol. Primary antibodies used were as follows: antitau CP13 (1:800), rabbit polyclonal to total tau (1:800), APP (1:800), and secondary antibodies against mouse and rabbit IgG conjugated to Alexa Fluor 564 (1:400) or Alexa Fluor 488 (1:400). Fixed cells were imaged with an inverted Zeiss LSM 780 confocal microscope using an oilimmersion objective $(40 \times / 0.55 \mathrm{NA})$. YFP fluorescence was excited by $488 \mathrm{~nm}$ line of the argon laser; the emission light was collected through the band-pass filter $(505-530 \mathrm{~nm})$. Red fluorescence (dsRED) was excited with the $\mathrm{He} / \mathrm{Ne}$ laser $(543 \mathrm{~nm})$ and the emission light was filtered with a long-pass filter with a cutoff $<560 \mathrm{~nm}$. Neuronal morphology was evaluated quantitatively with three parameters that describe neuron size and projection branching complexity. Briefly, the projection extension was estimated by measuring the sum of all the projections from images of single neurons imported in ImageJ after pixels were converted into micrometers. The skeletonized neuron length was estimated from total extensions and number of primary projections obtained. Branching complexity was investigated using the Sholl plug-in in Neuron-J from which the number of intersections along consecutive rings was obtained and represented in plots of projection intersection versus soma distance in micrometers. Statistical significance of data was determined with the two-tailed $t$ test. Values are expressed as mean \pm SEM.

\section{Results \\ Modulation of 3R:4R tau ratio by trans-splicing in differentiated human neurons}

Neuronal cultures were obtained by differentiation of hESCs (Hues9) using a standard protocol described previously (Fig. 1; Zhang et al., 2010). To test for proper polarization, immunofluorescence staining was performed $5 \mathrm{~d}$ after plating (DIV5); at this stage, human neurons showed early development of highly polarized structures with defined axonal (tau-positive) and dendritic (MAP2-positive) projections (Fig. 1B). After $19 \mathrm{~d}$ in culture (DIV19), enriched neuronal cultures displayed dense connectivity and axonal arborization together with detectable staining of tau in the axons (Fig. 1C). At this stage, neurons also show elec- 

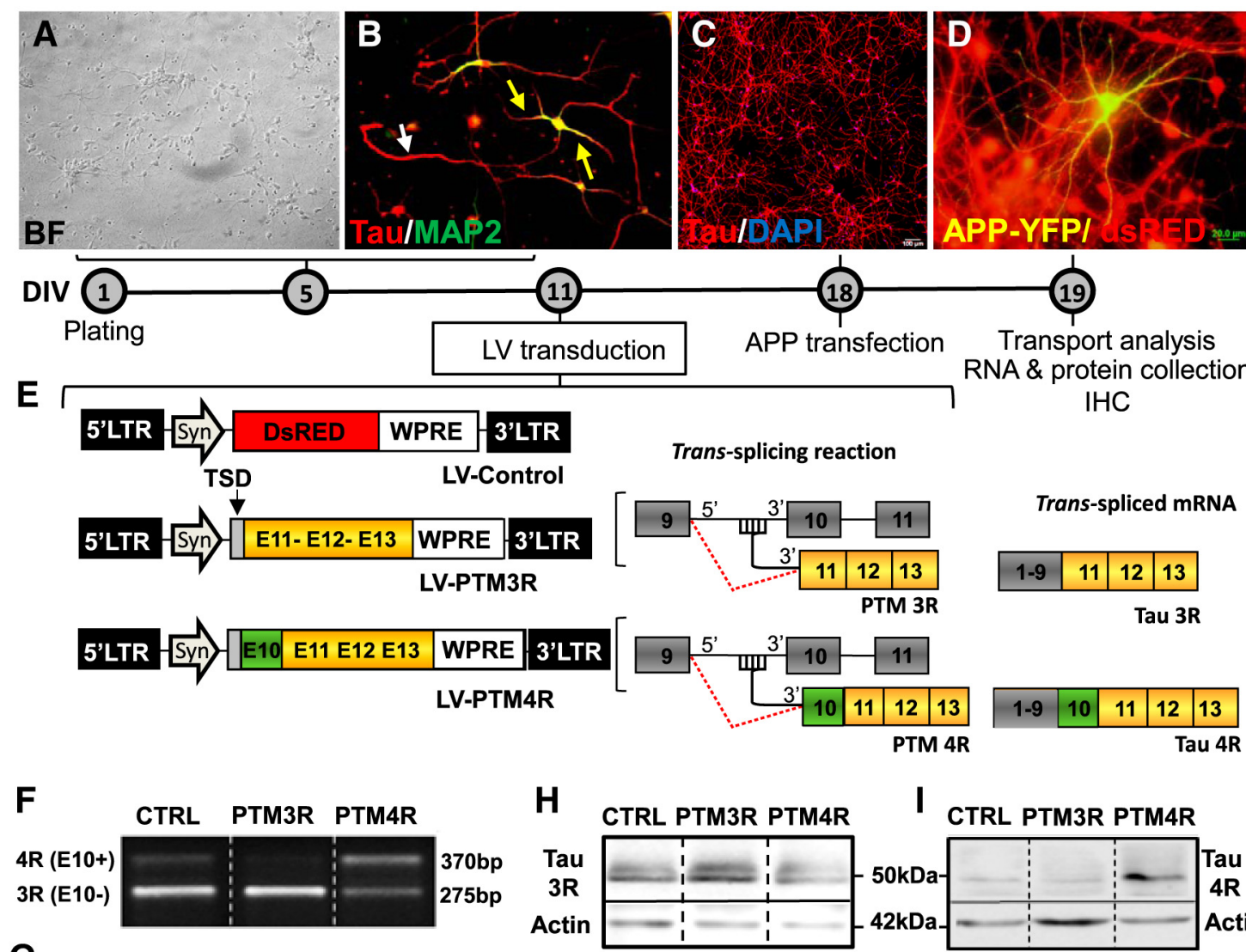

I CTRL PTM3R PTM4R
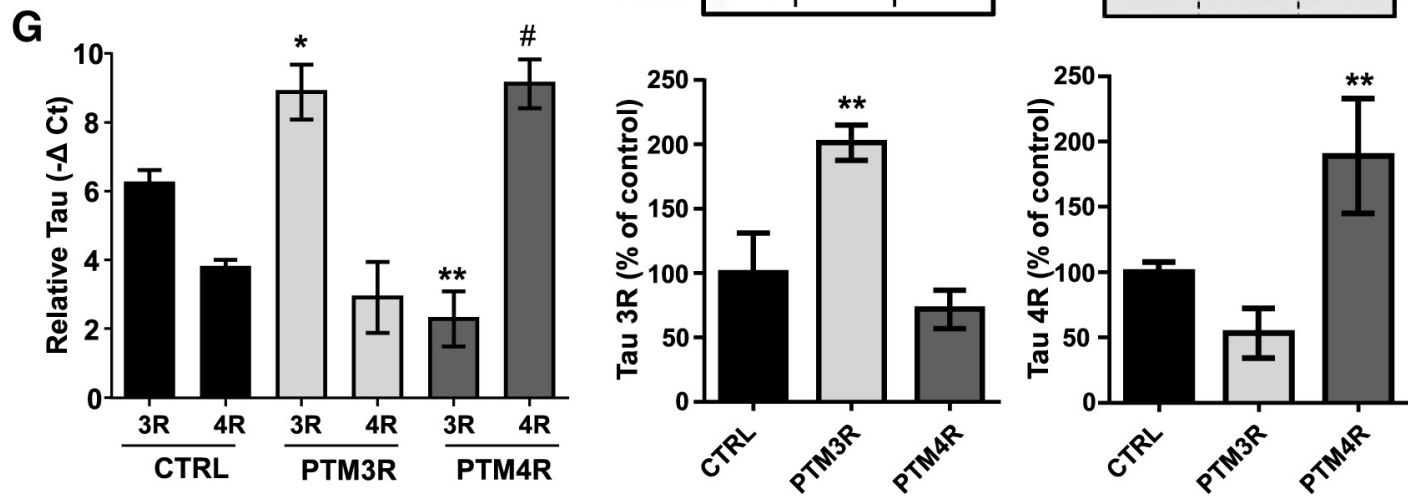

Figure 1. $3 R$ or $4 R$ tau modulation by trans-splicing in highly polarized human neurons. $A, B$, Human neuronal cultures at five days after plating (DIV5) showing projections and proper polarization in bright-field $(\boldsymbol{A})$ and after immunostaining with axonal and dendritic markers (tau and MAP2) (B). White arrow indicates the axon without MAP2 staining and yellow arrows show dendrites. C, Enriched human neuronal cultures at DIV18 showing highly polarized projections and dense connectivity. D, Neuron at DIV19 showing colocalization of APP-YFP (green) with LV-dsRED transduction (red). $\boldsymbol{E}$, Map of LVs used for neuronal transduction at DIV11. LV-dsRED: control vector carrying the reporter cassette; LV-PTM3R: LV carrying a pre-trans-splicing molecule with exons 11-13; or LV-PTM4R: with exons 10-13. TSD: trans-splicing domain consisting of a binding domain complementary to the 3' end of intron 9, a branch point and a 3' splice acceptor site. $\boldsymbol{F}$, Representative gel of RT-PCR products obtained with primers spanning tau exons $9-13$ simultaneously amplifying the $3 R$ and $4 R$ isoforms. RT-PCR was performed with mRNA extracted from neurons transduced with each LV. G, Relative content of 3 R and 4R tau isoforms determined by real time RT-PCR with specific primers for 3R (E10 -) and 4R (E10+) tau mRNA. Delta Ct was calculated relative to human ApoB gene expression (mean \pm SEM; $n=3$ ) in three independent experiments. One-way ANOVA followed by Dunnett's comparison versus control neurons performed separately for each isoform; ${ }^{*} p<0.05$ and ${ }^{* *} p<0.001$ versus $3 R$ CTRL; $\# p<0.001$ vs $4 R$ CTRL. $\boldsymbol{H}, \boldsymbol{I}$, Western blot analyses of human neuron homogenates. Shown is the detection of the $3 R(\boldsymbol{H})$ and $4 R(\boldsymbol{I})$ tau protein with isoform-specific antibodies normalized to actin quantified by optical density; ${ }^{* *} p<0.001(n=3)$; one-way ANOVA followed by Dunnett's comparison with control (CTRL) neurons (transduced with LV-dsRED).

trophysiological profiles of mature neurons, such as repetitive spiking and increased amplitude of voltage-dependent $\mathrm{Na}^{+}$and $\mathrm{K}^{+}$currents (Pozo Devoto et al., unpublished data).

To modulate the relative content of 3R:4R tau isoforms, we used a trans-splicing RNA reprogramming strategy (RodriguezMartin et al., 2005). Tau pre-trans-splicing molecules (PTMs) were delivered using LVs, as described previously (Avale et al., 2013). After integration into the neuron genome, the PTM cassette is transcribed into an RNA molecule that binds to intron 9 of the endogenous tau transcript and drives a trans-splicing reaction (Fig. 1E). We used two different LVs carrying Tau-PTMs: LVTau-PTM3R, containing exons $11-13$ to exclude exon 10 from the tau transcript, and LV-Tau-PTM4R, carrying exons $10-13$ to include exon 10 in the resulting trans-spliced product (Fig. 1E). Based on this strategy, LV-PTM3R is expected to increase the relative content of 3R tau, whereas LV-PTM4R would drive the production of $4 \mathrm{R}$ tau (Fig. 1E). High-density neuronal cultures were transduced at DIV11 with LV-Tau-PTM3R, LV-Tau- 

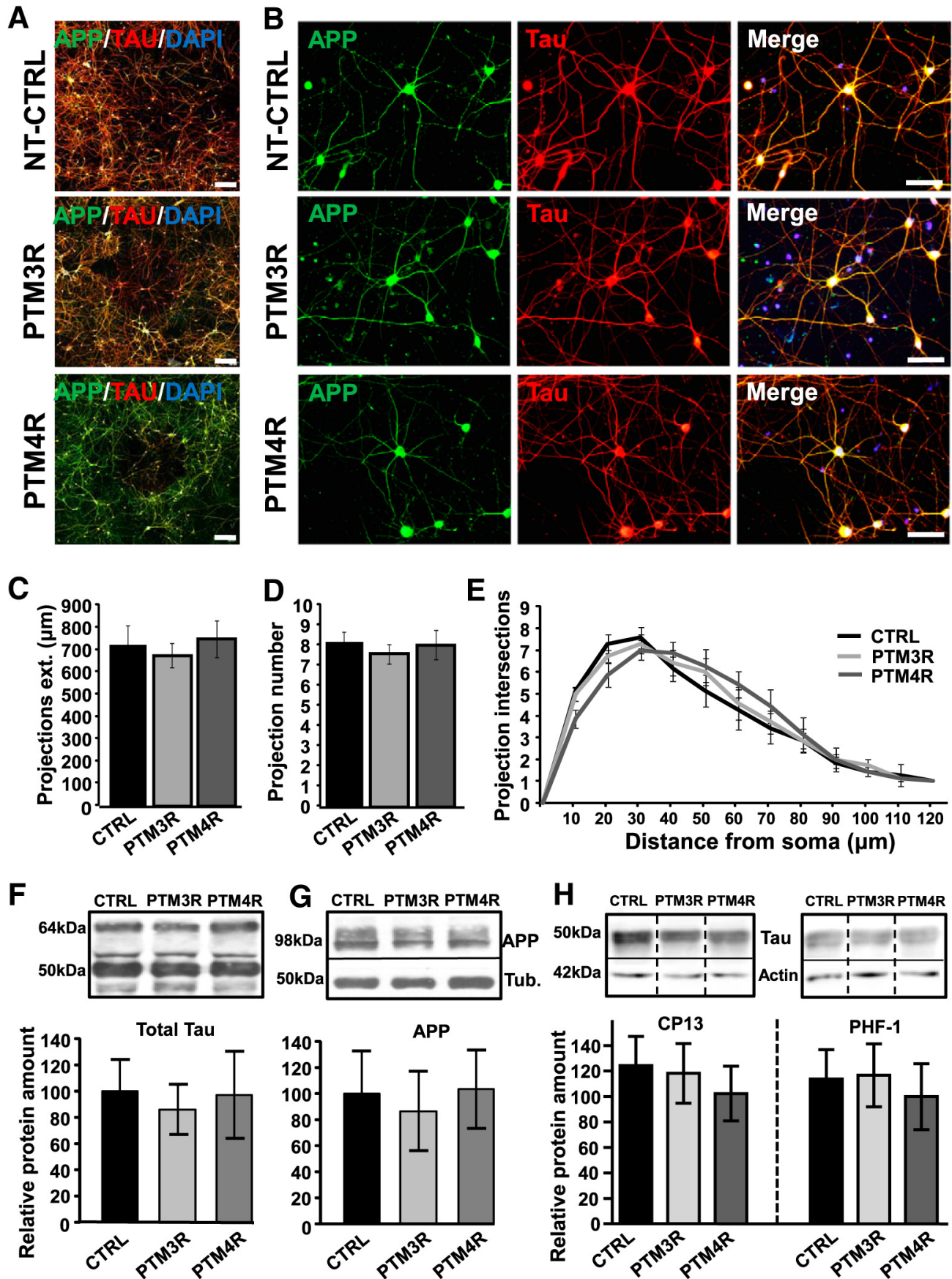

Figure 2. Normal neuronal polarization after 3R:4R tau isoform modulation. $A$, Confocal images of enriched neuronal cultures at DIV19 without transduction or transduced with LV-PTM3R or LV-PTM4R (from top to bottom). Immunofluorescence staining shows APP, tau, and Hoechst number for nuclei. $\boldsymbol{B}$, Confocal high-magnification images from control and transduced neurons stained for APP and tau showing polarization and extension integrity. $C, D$, Sum of neuronal projection extension expressed in micrometers $(H)$ and number of primary projection extensions from cell bodies ( I ) in neurons at DIV19 that were transduced at DIV11 with LV control, LV-PTM3R, and LV-PTM4R. E, Sholl analysis performed at DIV19 in transduced neurons showing the number of projection intersections versus distance from cell bodies (in micrometers). Data are shown as mean $\pm \mathrm{SEM} ; n=10) . \boldsymbol{F}, \mathbf{G}$, Western blot of neuronal homogenates showing total tau and APP protein levels after each LV transduction conditions compared with NTC neurons. Tubulin (tub) was used as a loading control. Data in $\boldsymbol{G}$ are expressed as percentage of NTC (mean $\pm S E M ; n=3$ ). $\boldsymbol{H}$, Western blot using antibodies specific to tau phosphorylated at Ser 202 (CP13) or Ser 396/404 (PHF-1). Quantitative analyses are related to $\beta$-actin signal as a loading control (mean \pm SEM; $n=3$ ).

PTM4R, or LV control, carrying a red fluorescent reporter gene (dsRED) instead of the PTM within the same vector backbone (Fig. 1E). Molecular, immunofluorescence and functional analyses were performed at DIV19 to allow maximal expression of the LVs. Transduction efficiency was assessed by the expression of the fluorescent reporter dsRED from the control LV (Fig. 1D).

To validate the trans-splicing system in human-derived neurons, we first analyzed $3 \mathrm{R}$ and $4 \mathrm{R}$ tau isoforms measuring the relative content of tau mRNA with or without E10 (hereafter also referred to as $4 \mathrm{R}$ and $3 \mathrm{R}$ mRNA, respectively; Fig. $1 F, G)$. End point RT-PCRs using primers that amplify both isoforms simultaneously from RNA of transduced neurons showed a clear change in tau isoforms relative content in either PTM transductions compared with control (Fig. $1 F$ ). We next performed a relative quantification analysis by real-time RT-PCR using isoform-specific primers to analyze changes in $3 \mathrm{R}$ and $4 \mathrm{R}$ relative 
A

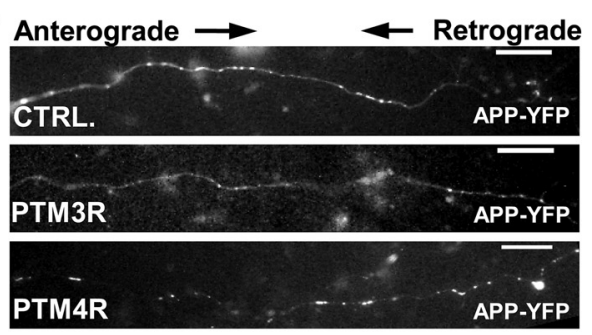

B

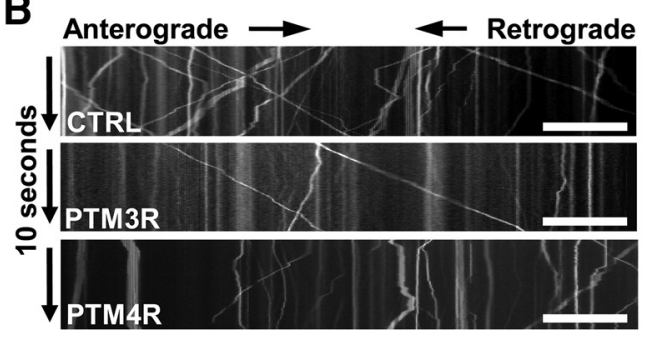

C
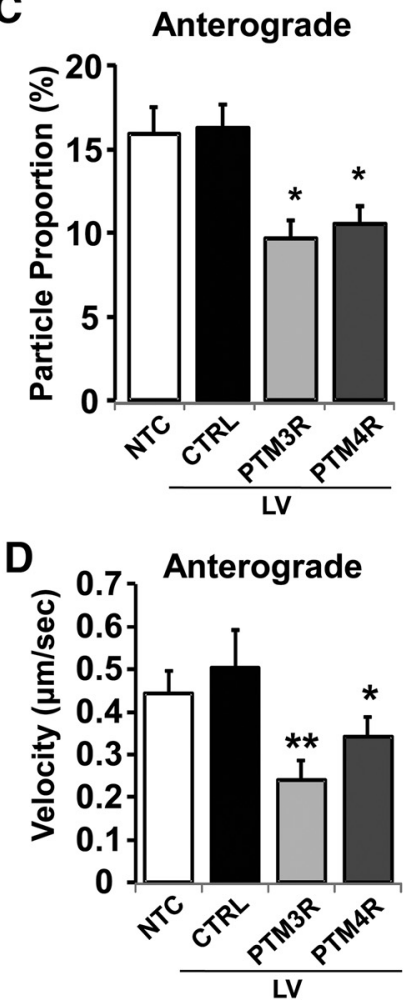
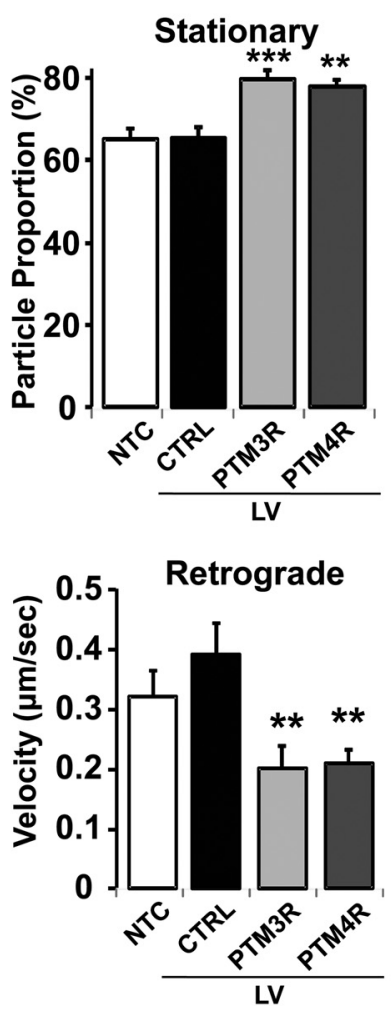

Retrograde

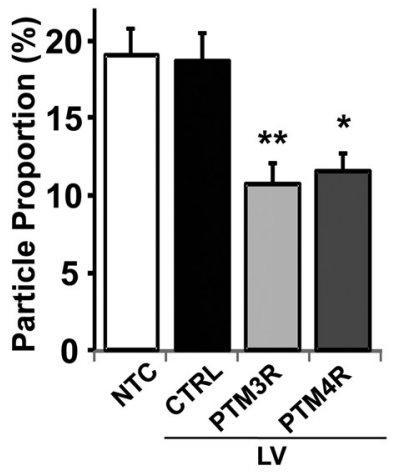

E

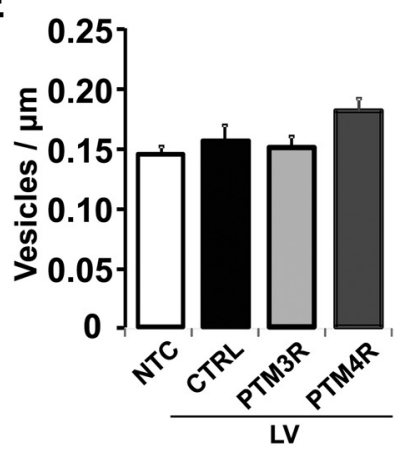

Figure 3. Impairment of axonal transport of APP vesicles in neurons under tau 3R:4R imbalance. $A$, Representative images from movies used to analyze the axonal transport of APP vesicles in neurons transduced with LV-control (dsRED), LV-PTM3R, and LV-PTM4R. Scale bar, $10 \mu \mathrm{m}$ (see Movies 1-3). $\boldsymbol{B}$, Kymographs of time versus distance from a 30 s movie generated at 8 frames/s in axons from neurons transfected with APP-YFP. Scale bar, $10 \mu \mathrm{m}$. C, Average proportion per kymograph of anterograde, stationary, and retrograde APP-YFP vesicles from NTCs or transduced with LV carrying dsRED, PTM3R, or PTM4R. D, Average velocity of anterograde and retrograde moving vesicles per kymograph. Kymographs analyzed: $n=59$ NTCs, $78 \mathrm{dsRED}, 57$ PTM3R, and 58 PTM4R. One-way ANOVA followed by Dunnett's comparison versus NTCs, ${ }^{*} p<0.05,{ }^{* *} p<0.02,{ }^{* * *} p<0.01$. E, Average number of APP-YFP vesicles per micrometer of axonal length in NTCs, dsRED, PTM3R, and PTM4R ( $n=25$ for each). One-way ANOVA was used for statistical analysis.

contents (Fig. $1 G$ and see Materials and Methods). Control neurons at DIV19 showed a $4 \mathrm{R} / 3 \mathrm{R}$ ratio of $0.6 \pm 0.07$, suggesting neuronal maturity evidenced by the presence of $4 \mathrm{R}$ tau, although the 3R:4R isoform balance was not yet achieved at this stage. As expected, transduction with LVs carrying either PTM3R or PTM4R induced a bias in the endogenous $4 \mathrm{R} / 3 \mathrm{R}$ ratio: LVPTM3R-transduced neurons showed a net $4 \mathrm{R} / 3 \mathrm{R}$ tau ratio reduction to $0.34 \pm 0.17$ due to a $30 \%$ relative increase in $3 \mathrm{R}$ tau, although the reduction observed in the $4 \mathrm{R}$ isoform was not statistically significant. Conversely, LV-PTM4R-transduced neurons showed a 2 -fold increase in $4 \mathrm{R}$ tau compared with control neurons, whereas a $50 \%$ reduction in $3 \mathrm{R}$ tau was observed, yielding a relative $4 \mathrm{R} / 3 \mathrm{R}$ ratio increase to $3.9 \pm 0.18$ (Fig. $1 G$ ).

To test whether the change in the relative content of tau isoforms observed at the RNA level was translated to the protein content, $3 \mathrm{R}$ and $4 \mathrm{R}$ tau were detected separately by Western blotting with isoform-specific antibodies in protein lysates obtained at DIV19 from each LV-transduced neuron (Fig. $1 H, I$ ). Due to potential differences in antibody affinities, it is not possible to estimate the $4 \mathrm{R} / 3 \mathrm{R}$ tau ratio accurately at the protein level, so changes in each tau isoform were analyzed independently, comparing PTM-transduced neurons and control conditions. Neurons transduced with LV PTM3R showed a significant increase in 3R tau protein amount compared with transduction with the control virus (Fig. $1 H$ ), with a mild reduction observed in this isoform under PTM4R transduction. Conversely, 4R tau protein content (Fig. 1I) was increased after PTM4R transduction and decreased in PTM3R-transduced neurons. Together, these results suggest that the trans-splicing strategy was efficient to modulate the relative content of tau isoforms in differentiated neurons both at the RNA and protein levels.

Normal neuronal polarization in human-derived neurons under 3R:4R tau isoform imbalance

We next evaluated whether changes in tau isoform ratio affects neuronal survival and/or morphology. Neuronal structure and polarization in transduced neurons were analyzed by immunofluorescence staining against cytoskeletal and membrane mark- 
ers at DIV19 (Fig. 2A,B). Tau and APP staining showed similar number of neurons in LV-PTM3R- and LV-PTM4R-transduced cultures compared with nontransduced controls (NTCs), displaying similar degree of ramification and arborization (Fig. $2 A, B)$. In addition, no changes in the number of projections or differences in axonal or dendrite length were observed between control and LV-PTM3R- or LV-PTM4R-transduced neurons (Fig. 2C-E). Transduced neurons did not show evidence of axonal retraction or swelling or accumulation of tau in their projections (Fig. $2 B$ ). These results indicate that neither the transduction with LVs nor the induced changes in tau isoforms ratio alter survival, polarization, or morphology of human neurons in culture.

To test whether PTM expression, or the LV transduction, alter the amount of total tau protein we performed Western blot analysis from homogenates collected at DIV19 using an anti-tau or anti-APP antibody. No changes in the amount of total tau or APP were observed among control (dsRED), LV-PTM3R-, and LV-PTM4R-transduced neurons (Fig. $2 F, G$ ), showing that neither LV transduction nor the modulation of tau isoforms alters the total content of these proteins. To test whether a shift in tau isoforms balance might induce changes in tau phosphorylation, we also performed Western blot analyses using antibodies recognizing tau phosphorylated at Ser202 (CP13) or at Ser 396/404 (PHF1). No differences were observed among the experimental conditions, suggesting that the level of tau phosphorylation is not affected after trans-splicing (Fig. $2 H$ ). Therefore, through the use of the trans-splicing strategy, we have been able to modulate the relative 3R:4R tau ratio in differentiated human neurons without altering tau protein expression, phosphorylation state, neuronal morphology, or survival. We thus reasoned that this is a suitable model with which to investigate particular neuronal functions such as axonal transport that might be affected by tau isoform imbalance.

\section{APP axonal transport impairment after changes in the 3R:4R tau ratio}

After determining that the 3R:4R tau ratio can be modulated efficiently by trans-splicing, we next investigated whether imbalance in tau isoforms perturbs APP axonal transport. To answer this question, APP dynamics was monitored at DIV19 by continuous high-definition live-imaging recordings to generate movies from axons identified by morphology, as described previously (Otero et al., 2014). Neurons were transduced at DIV11 with LV-PTM3R, LV-PTM4R, or control LV-dsRED and transfected at DIV18 with the fluorescent hAPP695-YFP expression vector (pCDNA3-APP-YFP) to track fluorescent APP vesicles, as indicated in the experimental timeline (Fig. 1). Colocalization of YFP and dsRED signals in transduced controls showed that $99 \%$ of APP-YFP-transfected cells were also transduced with the LV (Fig. 1D). A group of neurons that had not been transduced with LVs (NTCs) was also included in the experiment to rule out any effect over APP transport due to LV transduction. Movies were obtained from axons at their middle part (at least $200 \mu \mathrm{m}$ away from the cell body or tip) to generate detailed kymographs of time versus distance for qualitative and quantitative analyses (Fig. $3 \mathrm{~A}$ and see Movies 1, 2, and 3). The parameters analyzed (velocities and run lengths) of APP dynamics in control (NTC) neurons were comparable to those described for different neuronal settings from mouse, chicken, and Drosophila, with vesicular movement undergoing fast anterograde and retrograde axonal transport (Goldsbury et al., 2007; Falzone and Stokin, 2012; Reis et al., 2012). NTC human neurons revealed an even distribution

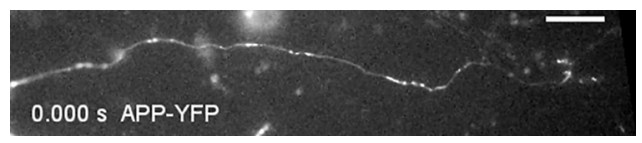

Movie 1. APP transport in a control neuron. This is a representative movie showing the axonal transport of APP vesicles in real time in a neuron that has been transduced with control lentivirus and transfected with APP-YFP. Movie showing time in seconds ( 8 frames $/ \mathrm{s}$ ) is oriented so that right- to left-moving vesicles correspond to anterograde transport and left to right to retrograde movement. Scale bar, $20 \mu \mathrm{m}$.

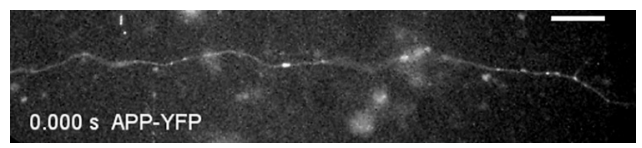

Movie 2. APP transport in PTM3R-transduced neuron. This is a representative movie showing axonal transport of APP vesicles in real time in a neuron that has been transduced with PTM3R lentivirus and transfected with APP-YFP. Movie showing time in seconds ( 8 frames $/ \mathrm{s}$ ) is oriented so that right- to left-moving vesicles correspond to anterograde axonal transport and left to right to retrograde movement. Scale bar, $20 \mu \mathrm{m}$.

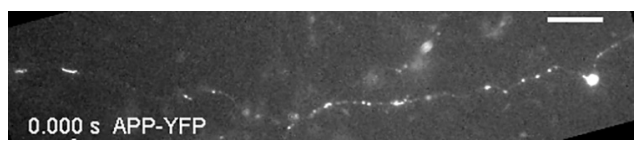

Movie 3. APP transport in PTM4R-transduced neuron.: This is a representative movie showing the axonal transport of APP vesicles in real time in a neuron that has been transduced with PTM4R lentivirus and transfected with APP_YFP. Movie showing time in seconds (8 frames/s) is oriented so that right- to left-moving vesicles correspond to anterograde axonal transport and left to right to retrograde movement. Scale
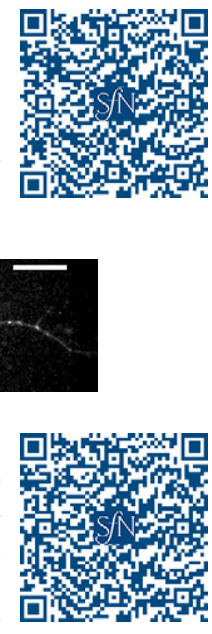
bar, $20 \mu \mathrm{m}$

of anterograde- and retrograde-moving APP vesicles and a fraction of stationary vesicles under the time frame analyzed (Fig. 3C). The average velocity for anterograde- and retrogrademoving APP vesicles in NTC conditions (Fig. 3D) were consistent with the values obtained for fast axonal transport in other systems (Stokin et al., 2005; Falzone et al., 2009).

Transduction with either LV-PTM3R or LV-PTM4R impaired the axonal transport of APP significantly (Fig. 3C). More specifically, increased levels of the 3R isoform (by LV-PTM3R transduction) led to $30-50 \%$ reductions of anterograde and retrograde APP movement, respectively, and significantly increased the proportion of stationary vesicles by $30 \%$ (Fig. 3C). Interestingly, shifting the balance toward the $4 \mathrm{R}$ isoform (LV-PTM4R transduction) produced a similar reduction of the APP anterograde and retrograde transport while increasing the stationary proportion (Fig. $3 C$ ). The density of APP-YFP vesicles in axons was similar between the different conditions (Fig. $3 E$ ), revealing that the observed reduction in transport is likely to be induced by a shift from moving toward stationary vesicles. When analyzing the dynamics of net moving vesicles of APP-YFP, anterograde and retrograde average velocities (including movement and pauses of each analyzed particle) were significantly reduced in LV-PTM3R- and LV-PTM4R-transduced neurons compared with dsRED or NTC neurons (Fig. 3D). Control transduced (LV-dsRED) neurons showed similar proportions and average velocities of APP-YFP vesicles compared with control NTC neurons, suggesting that LV transduction itself does not modify APP axonal transport properties measured $8 \mathrm{~d}$ after transduction (Fig. 
A

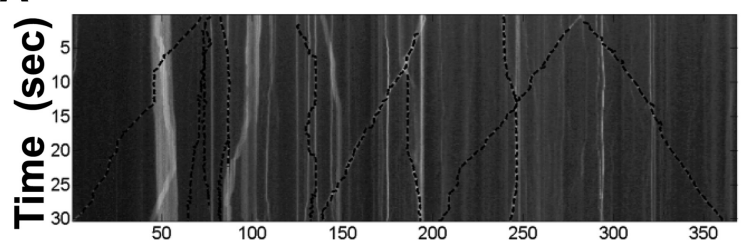

Distance $(\mu \mathrm{m})$

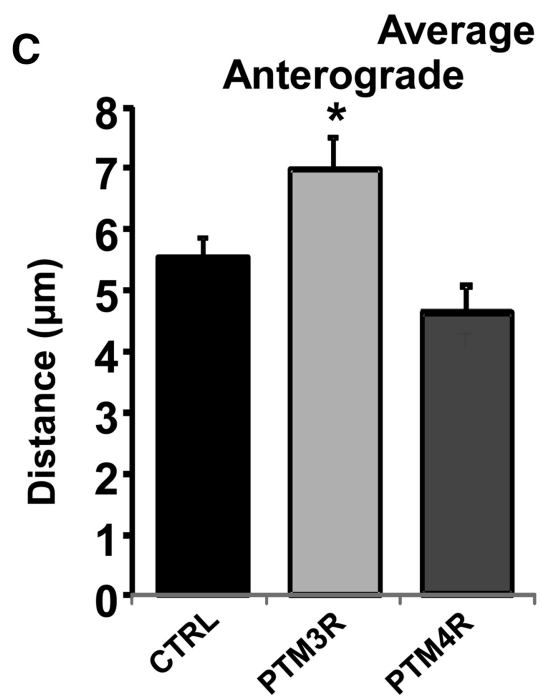

E

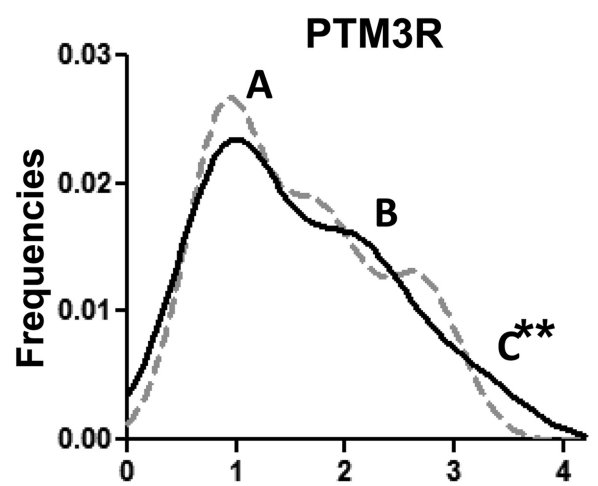

G

G Segmental velocities ( $\mu \mathrm{m} / \mathrm{sec})$

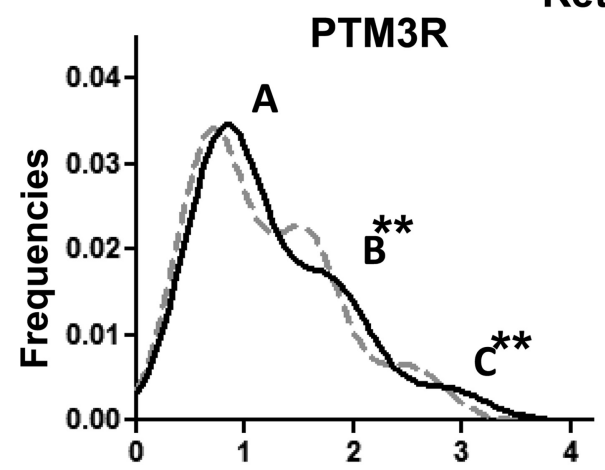

Segmental velocities $(\mu \mathrm{m} / \mathrm{sec})$
B

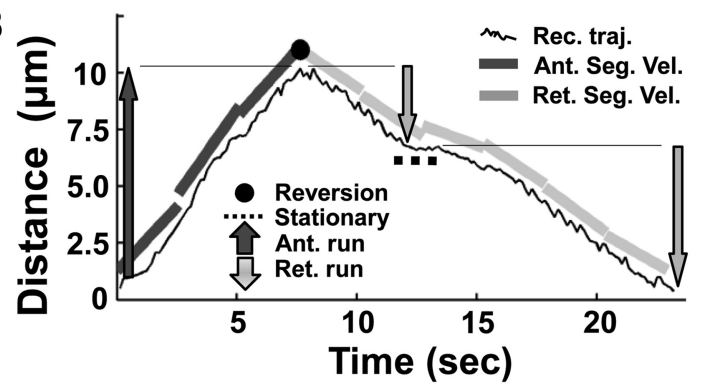

Retrograde

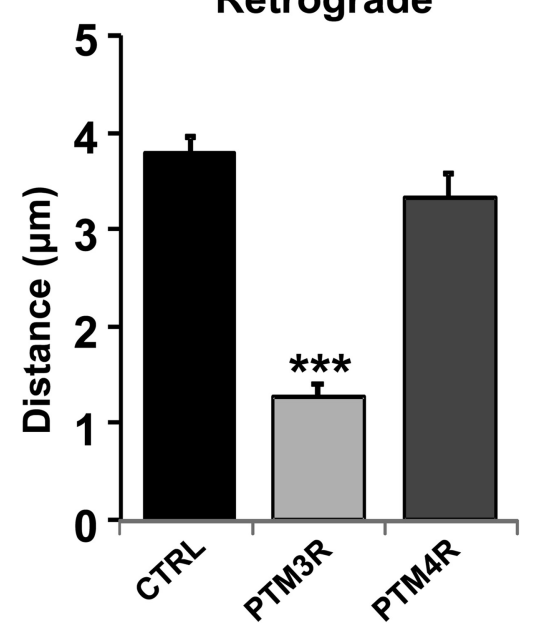

Anterograde
D 4 Pauses

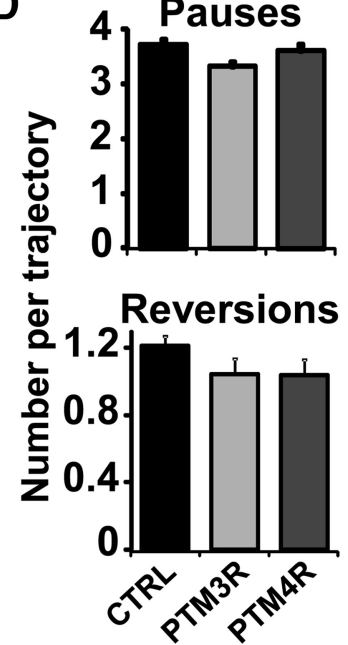

F
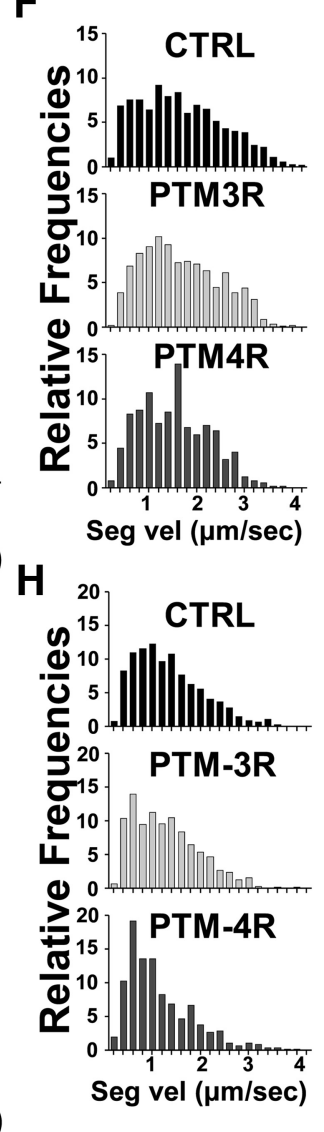

Figure 4. Kinesin and dynein transport properties are differentially impaired by tau isoform ratio modulation in human neurons. $\boldsymbol{A}$, Representative kymographs of time versus distance showing moving and stationary APP vesicles and their trajectories using MATLAB (dotted lines). $\boldsymbol{B}$, Representative recovered trajectory plotted as a function of distance versus time showing the properties extracted using custom-made MATLAB scripts. $\boldsymbol{C}-\boldsymbol{E}$, Run lengths, segmental velocities, pauses, and reversions were determined from a total of 1283 (Figure legend continues.) 
$3 C, D)$. Together, these results showed significant changes in the movement of APP vesicles when the endogenous ratio of 3R:4R tau isoforms is modified, suggesting that axonal transport of APP is tightly regulated by the ratio of tau isoforms either attached to MTs or soluble in the axonal shaft.

\section{Differential anterograde and retrograde regulation over APP axonal transport by $3 \mathrm{R}$ or $4 \mathrm{R}$ tau isoforms}

To further understand tau regulation over APP transport dynamics in human neurons and to investigate potential mechanisms affected by the 3R:4R imbalance, fluorescent APP moving vesicles were tracked using an algorithm developed to identify particle positions with subpixel precision. Single trajectories obtained from movies (Fig. $3 A, B$ ) were analyzed using custom-made MATLAB routines to extract axonal transport properties such as run length, number of pauses per trajectories, reversion of directionality, and segmental velocity distributions (Fig. 4 and see Materials and Methods). To understand whether tau can regulate processive axonal transport of APP, we computed the anterograde average run length of APP vesicles in LV-PTM3R- and LV-PTM4R-transduced neurons and compared them with control transduced neurons (Fig. 4C). Interestingly, shifting tau isoforms toward 3R content (LV-PTM3R) induced a significant $(30 \%)$ increase of the anterograde run length, but enhancing $4 \mathrm{R}$ isoform (LV-PTM4R) showed no significant effect on anterograde runs (Fig. 4C). Conversely, LV-PTM3R transduction induced a significant effect on the retrograde component with $>70 \%$ reduction in the average run length, whereas LV-PTM4R showed no changes over the average retrograde run length compared with control conditions (Fig. 4C). We did not observe significant changes in the number of pauses within trajectories and in reversion from anterograde to retrograde transport or vice versa due to tau 3R:4R shifting (Fig. 4D). Next, we analyzed the distribution of segmental velocities of moving APP vesicles in LV-PTM3R- and LV-PTM4R-transduced compared with control transduced neurons (Fig. 4E,F). A multimodal distribution of segmental velocities was observed, so we propose a Gaussian mixture model with three components to describe each dataset (see Materials and Methods and Reis et al., 2012). The distribution of anterograde segmental velocities in control transduced neurons ranges from 0.1 to $5 \mu \mathrm{m} / \mathrm{s}$ and can be adjusted to modes A-C (Fig. 4E). A similar result was obtained for the distribution of retrograde segmental velocities, but with more probable lower velocities (Fig. 4F). The distributions obtained from neurons transduced with either LV-PTM3R or LV-PTM4R showed that anterograde segmental velocities are affected by shifting tau ratios

\section{$\leftarrow$}

(Figure legend continued.) trajectories (see Materials and Methods for details). C, Anterograde and retrograde average run lengths of vesicles moving in a net direction obtained from APP-YFP trajectories in neurons transduced with LV-dsRED (control), PTM3R, or PTM4R. $n=$ ctrl: antero 1551, retro 1548; PTM3R: antero 572, retro 555; PTM4R: antero 465, retro 503. One-way ANOVA followed by Dunnett's comparison versus Ctrl ${ }^{*}<0.05,{ }^{* * *}<0.01$. D, Average number of pauses and reversions per trajectory. Pauses $n=$ ctrl: 2752; PTM3R: 981; PTM4R: 913. Reversion: $n=$ ctrl: 895; PTM3R: 307; PTM4R: 262. $\boldsymbol{E}-\boldsymbol{H}$, Distribution of segmental velocities of moving APP-YFP vesicles. A Gaussian mixture model with three modes $(\boldsymbol{A}-\boldsymbol{C})$ was used to represent the segmental velocities distributions for anterograde $(\boldsymbol{E})$ and retrograde $(\boldsymbol{G})$ APP vesicle transport in control (full line) or transduced with PTM3R (light gray) or PTM4R (dark gray) LVs (dotted lines). The center and fraction of the different modes are displayed in Table 1. Relative frequencies of anterograde $(\boldsymbol{F})$ and retrograde $(\boldsymbol{H})$ segmental velocities used for model construction. Segmental velocities $n=$ ctrl: antero 1671, retro 1344; PTM3R: antero 795, retro 549; PTM4R: antero 916, retro 752. Significant differences $\left({ }^{* *}\right)$ obtained from comparison of nonoverlapping confidence intervals with the control group (LV-dsRED transduced neurons).
Table 1. Segmental velocity data modeled as a combination of normal distributions

\begin{tabular}{|c|c|c|c|c|c|}
\hline \multicolumn{6}{|l|}{ Anterograde } \\
\hline Mode center & \multicolumn{2}{|c|}{ A Max \pm error } & \multicolumn{2}{|c|}{ B Max \pm error } & C Max \pm error \\
\hline PTM-dsRED & \multicolumn{2}{|c|}{$0.94 \pm 0.07$} & \multicolumn{2}{|c|}{$2.09 \pm 0.19$} & $3.11 \pm 0.38$ \\
\hline PTM-3R & \multicolumn{2}{|c|}{$0.90 \pm 0.04$} & \multicolumn{2}{|c|}{$1.77 \pm 0.09$} & $2.69 \pm 0.06^{* *}$ \\
\hline PTM-4R & \multicolumn{2}{|c|}{$0.78 \pm 0.07^{* *}$} & \multicolumn{2}{|c|}{$1.62 \pm 0.13^{* *}$} & $2.49 \pm 0.16^{* *}$ \\
\hline Mode fraction & A Mode & B Mode & CMode & Error & Sigma \pm error \\
\hline PTM-dsRED & 0.54 & 0.34 & 0.12 & \pm 0.05 & $0.24 \pm 0.04$ \\
\hline PTM-3R & 0.47 & 0.31 & 0.23 & \pm 0.03 & $0.13 \pm 0.02$ \\
\hline PTM-4R & 0.42 & 0.39 & 0.19 & \pm 0.05 & $0.12 \pm 0.03$ \\
\hline
\end{tabular}

Retrograde

\begin{tabular}{|c|c|c|c|c|c|}
\hline Mode center & \multicolumn{2}{|c|}{ A Max \pm error } & \multicolumn{2}{|c|}{ B Max \pm error } & C Max \pm error \\
\hline PTM-dsRED & \multicolumn{2}{|c|}{$0.81 \pm 0.03$} & \multicolumn{2}{|c|}{$1.78 \pm 0.09$} & $2.88 \pm 0.14$ \\
\hline PTM-3R & \multicolumn{2}{|c|}{$0.69 \pm 0.04$} & \multicolumn{2}{|c|}{$1.55 \pm 0.09^{* *}$} & $2.52 \pm 0.14^{* *}$ \\
\hline PTM-4R & \multicolumn{2}{|c|}{$0.71 \pm 0.02$} & \multicolumn{2}{|c|}{$1.78 \pm 0.10$} & $3.04 \pm 0.18$ \\
\hline Mode fraction & A Mode & B Mode & C Mode & Error & Sigma \pm error \\
\hline PTM-dsRED & 0.64 & 0.29 & 0.07 & \pm 0.03 & $0.14 \pm 0.01$ \\
\hline PTM-3R & 0.55 & 0.35 & 0.10 & \pm 0.04 & $0.11 \pm 0.02$ \\
\hline PTM-4R & 0.72 & 0.23 & 0.05 & \pm 0.02 & $0.11 \pm 0.01$ \\
\hline
\end{tabular}

Shown are the values of the central position and fraction of the modes parameters of a Gaussian mixture model with three components $(A, B$, and $C$ ) obtained using an expectation maximization algorithm (bold). Errors were determined as the SD of $n=1000$ resampling with replacements bootstrapping procedure. ${ }^{* *}$ Significant differences obtained from comparison of nonoverlapping confidence intervals with control group (LV-dsRED transduced neurons).

either toward 3R or 4R (Fig. 4E, F, Table 1). Enhanced 3R content showed mild shape changes in the anterograde segmental velocity distribution, revealing reduced modes of velocities mainly in the $\mathrm{C}$ mode with enhanced representation for mode A (Fig. 4E, Table $1)$. Conversely, shifting tau content toward $4 \mathrm{R}$ induced significant reductions in anterograde velocities for modes A-C compared with control and with LV-PTM3R transduction (Fig. 4E). Under 3R increase, the amplitude of anterograde modes A and $\mathrm{B}$ were significantly increased, suggesting also a shift in the total distribution to lower anterograde velocities (Fig. 4E, Table 1). Retrograde axonal transport of APP under enhanced 3R isoform showed significant changes of retrograde segmental velocity distributions compared with control (Fig. 4F). Nevertheless, no change in the number of segmental velocities representing each mode pick (mode weight) was observed in LV-PTM3Rtransduced neurons (Fig. 4F, Table 1), suggesting that increases in $3 \mathrm{R}$ tau induce mild reductions in the dynein movement, affecting the retrograde component. Neurons transduced with LV-PTM4R showed less impact on the retrograde movement with similar retrograde modes compared with control; however, enhanced mode weights were observed for mode A, with significant weight reductions for modes B and C (Fig. 4F, Table $1)$. Together, these results suggest that changes in the tau isoform ratio toward increases either in $3 \mathrm{R}$ or $4 \mathrm{R}$ can modulate the properties of anterograde and retrograde APP vesicle movement directly by differentially changing the run length and modifying the distribution of segmental velocities of moving vesicles.

\section{Discussion}

Here, we tested whether relative content in tau isoforms played crucial roles in the regulation of axonal transport, combining the RNA trans-splicing strategy with high-definition/high-speed live imaging of APP vesicles in human-derived neurons. Our findings have particular relevance to understanding early neuronal dys- 
function and the interplay between tau and APP in molecular pathways of disease (Götz et al., 2006; Goldstein, 2012).

Axonal transport is one of the tau-regulated mechanisms likely perturbed in tauopathies, yet the available data seem controversial. Several experimental models showed axonal cargo depletion, clogging, and transport defects after tau overexpression (Ebneth et al., 1998; Stamer et al., 2002; Mertens et al., 2013). Moreover, impairments in transport dynamics have been observed under abnormal tau 3R:4R content in cultured neurons (Mertens et al., 2013; Iovino et al., 2015) and in mouse models (Dawson et al., 2007). However, tau overexpression or diseaseassociated mutants might have underlined some of the observed phenotypes, making it difficult to determine the precise role of tau isoform ratio in transport. Conversely, some experiments showed no defects in ${ }^{35} \mathrm{~S}$-methionine-labeled protein distribution in tau knock-out mouse models, suggesting that tau deletion or overexpression might not influence transport (Yuan et al., 2008; Yuan et al., 2013). However, it has been shown that tau knock-out mice upregulate other MAPs as a compensatory mechanism (Ke et al., 2012; Ma et al., 2014), which may account for the lack of transport deficits in those mice. Furthermore, it is particularly difficult to analyze the role of 3R:4R tau balance in mice because only $4 \mathrm{R}$ tau is expressed in the adult rodent brain. The trans-splicing strategy have created a unique platform to dissect the functional consequences of human tau isoform imbalance, allowing the modulation of the endogenous tau isoform ratio in differentiated human neurons without altering total tau protein or impairing other tau-dependent functions such as axon elongation or neuronal polarization.

Axonal transport defects underlie many neurological diseases (Stokin et al., 2005; De Vos et al., 2008; Goldstein, 2012). However, it remains unclear whether such impairments represent an early causative effect or a late consequence in disease (Götz et al., 2006; Goldstein, 2012; Bodea et al., 2016). Particularly, the transport of APP and its subcellular mislocalization have putative implication in Alzheimer's disease, with tau playing a crucial role in controlling APP trafficking (Stamer et al., 2002; Goldsbury et al., 2007). Here, we show that changes in the endogenous $3 R: 4 R$ tau ratio are sufficient to impair the transport of APP vesicles significantly in human neurons, suggesting a possible pathological link between tau isoform imbalances and APP. These results open new avenues to understand how tau isoforms modulate, not only the dynamics, but also the metabolism of APP, which is intimately linked to its intracellular transport (Goldstein, 2012).

From a mechanistic perspective, our data complement previous evidence about the role of tau in the regulation of molecular motors. Two models have been proposed for the bidirectional transport of cargos such as APP vesicles along MTs. The coordination model assumes that anterograde kinesin and retrograde dynein motors activity are regulated by accessory proteins, and thus the direction of motion depends on which motor is active (Leidel et al., 2012; Fu and Holzbaur, 2013). The tug-of-war model postulates that cargo direction is exerted by the larger net force resulting from both polarity motors simultaneously active (Müller et al., 2008). Recently, a hybrid interaction between both models was proposed to lead transport (Hendricks et al., 2012). The many physiological roles of tau can modulate transport by regulating the motor-MT interaction (Trinczek et al., 1999; Magnani et al., 2007), modifying the MT structure and/or triggering phosphorylation cascades that modify motors (Kanaan et al., 2012). In this context, we propose that APP vesicles are bound to multiple kinesin and dynein motors that are regulated by tau (Fig. 5). Tau binding transiently to MTs increases the probability of
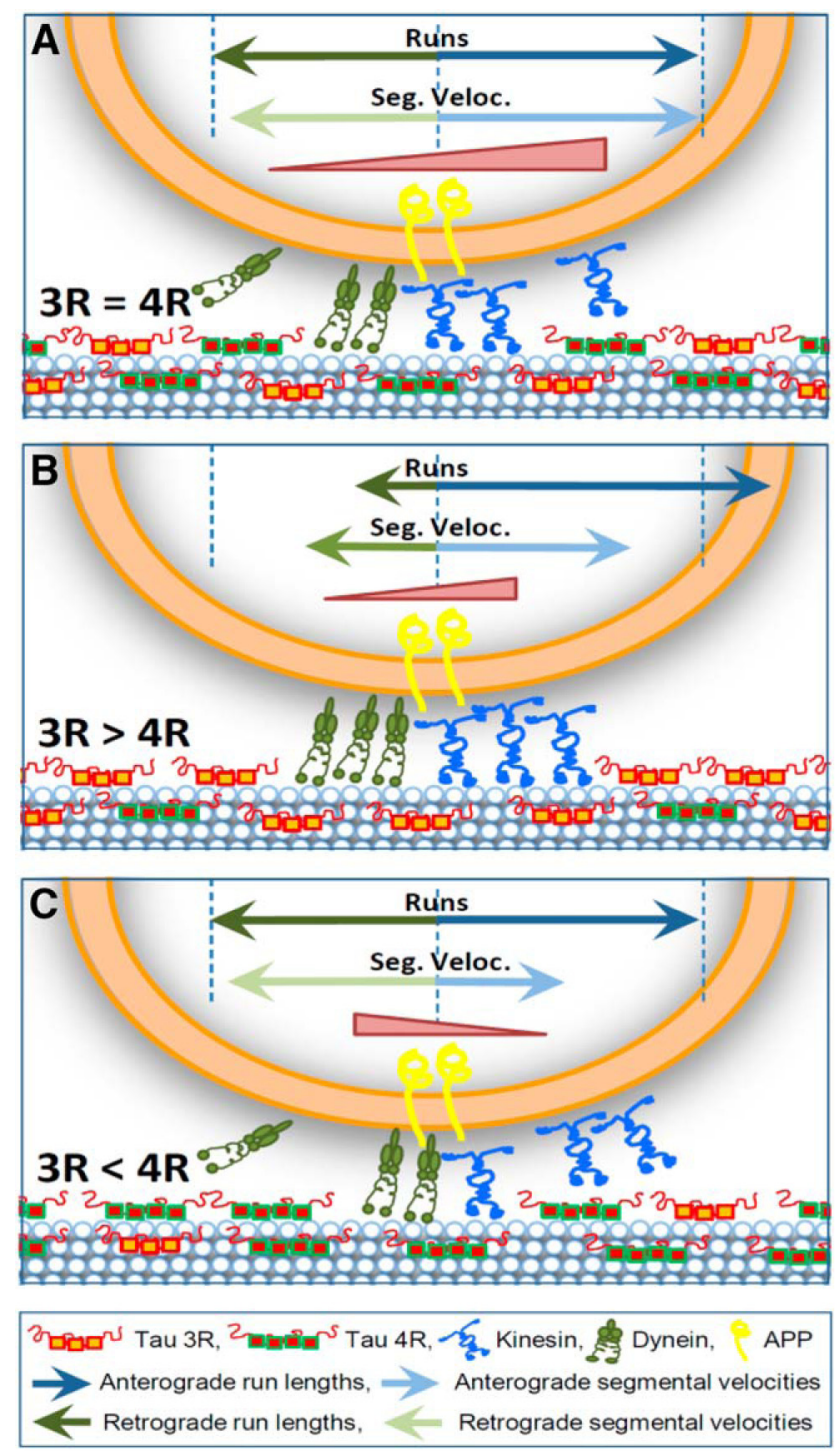

Figure 5. Hybrid competition-regulation model of tau-isoform-dependent APP axonal transport. We propose a model in which the APP vesicle is driven by two groups of motors, kinesin and dynein, along tau-decorated MTs. Motors behave in a tug-of-war/coordination scenario, displaying a differential tau isoform-dependent interaction with MTs. $A$, In the control condition, the two groups of motors are balanced to result in higher anterograde runs (dark arrows) and segmental velocities (light arrows) compared with the retrograde ones, giving an anterograde bias (red triangle) for the movement of APP vesicles. $\boldsymbol{B}$, Shifts in the ratio toward $3 R$ would not change the amount of tau bound to MT, but rather would reduce its binding strength, facilitating the recruitment of extra active motors. Extra kinesin load leads to an anterograde bias (red triangle) that favors the distal delivery of APP vesicles. C, Increased 4R tau level induces a higher binding strength of tau to MT that facilitates anterograde motor detachment and strongly impairs kinesin velocities (light arrows), whereas no significant changes in retrograde runs or velocities are observed. This results in a retrograde bias (red triangle) that should favor retrieval of APP vesicles to the cell body.

kinesin detachment (Dixit et al., 2008) and, if the tau concentration is high, the probability of unbound kinesin to attach to the MT is reduced (Vershinin et al., 2007; Trinczek et al., 1999). Conversely, dyneins are less affected by tau on MT (Dixit et al., 2008). Therefore, we propose that, under balanced content of 3R:4R tau, few weak dyneins and stronger kinesins would transport the APP vesicle, resulting in control values of run lengths and segmental velocities (Fig. 5A). Based on binding results showing that the affinity of $3 \mathrm{R}$ isoform for MTs is lower than that of $4 \mathrm{R}$ (Goode et al., 2000) and assuming that affinity is directly 
related to the mean attachment time of tau on a MT, we propose that the $3 \mathrm{R}$ increase enhances the number of active motors driving the cargo, although the number of sites occupied by tau within the MT would remain equal and the engagement of extra kinesins would lead to enhanced anterograde processivity (longer runs). The reduction in retrograde runs and segmental velocities in $3 \mathrm{R}$ are also consistent with this model because kinesin could act as a dynein anchor (Fig. 5B). Conversely, $4 \mathrm{R}$ increase would facilitate motor detachment and impair kinesin (Trinczek et al., 1999). This is consistent with the reduction in the anterograde velocities observed in this condition, suggesting a reduced load in the number of active kinesins driving the APP vesicle with no change in runs (Fig. 5C). A priori, this mechanistic model is consistent with in vitro experiments showing that kinesin activity is highly regulated by tau compared with dynein (Vershinin et al., 2007; Dixit et al., 2008; McVicker et al., 2011). However, there is an apparent contradiction between our experimental data and previous reports showing that long $4 \mathrm{R}$ tau is less inhibitory for single kinesin than short $3 \mathrm{R}$ tau, which is proposed to be due to an increased static inhibitory state of short 3R in artificially stabilized MTs (Dixit et al., 2008; McVicker et al., 2014). However, such differential impact on kinesin could be explained by the different number of $\mathrm{N}$-terminal repeats in the tau variants used in those studies (short tau $=0 \mathrm{~N} 3 \mathrm{R}$ and long tau $=2 \mathrm{~N} 4 \mathrm{R}$ ). $\mathrm{N}$-terminal repeats also modulate kinesin velocities; being $0 \mathrm{~N}$ more inhibitory than $2 \mathrm{~N}$ and $3 \mathrm{R}$ less inhibitory than $4 \mathrm{R}$ (Tarhan et al., 2013). In our system, $3 \mathrm{R}$ and $4 \mathrm{R}$ tau are not expected to differ in the $\mathrm{N}$-terminal repeats given that the trans-splicing reaction occurs at intron 9 spatiotemporally downstream of exons 2 and 3 splicing.

Although we propose a simplified model of tau isoforms affecting motors through their differential binding to MTs (Fig. 5), it is noteworthy that such regulation of transport could also arise indirectly upon changes in other tau isoform-dependent functions such as the regulation of MT dynamics (Stamer et al., 2002), motor stability (Tarhan et al., 2013), the interaction with the dynactin complex (Magnani et al., 2007), and/or the control of kinases involved in the phosphorylation of motors (Kanaan et al., 2012).

Finally, these findings also highlight the relevant physiological role of tau alternative splicing in the human brain. During early development, all mammals express high levels of $3 \mathrm{R}$ tau in the nervous system; however, whereas $4 \mathrm{R}$ tau is predominant in the adult brain of most species, the (normal) adult human brain contains equal levels of 3R and 4R tau (Goedert et al., 1989; Andreadis, 2005). A differential anterograde to retrograde bias for $3 \mathrm{R}$ and $4 \mathrm{R}$ tau in axonal transport is consistent with the predominance of $3 \mathrm{R}$ tau during neuronal development that should favor the distal delivery of cargos in projections that need to grow and extend, whereas the 3R:4R balance reached in mature human neurons would be functional to maintain synapse delivery but also retrieval of survival factors to the soma. The human neurons derived from hESCs used in this study displayed an endogenous $4 \mathrm{R} / 3 \mathrm{R}$ ratio of 0.6 after $19 \mathrm{~d}$ in culture, showing that the $3 \mathrm{R}$ isoform still predominates over $4 \mathrm{R}$, probably due to the embryonic origin of the neurons and the short time in culture. The trans-splicing induced by LV-PTM3R or LV-PTM4R creates an imbalance of tau isoforms, leading to significant shifts in the almost equivalent ratio of human-derived neurons. Changes in the relative content of $3 R$ and $4 R$ tau have been shown to affect MT assembly and might impair proper neuronal polarity development (Panda et al., 2003). However, we did not observe significant changes in neuronal morphology or polarization after trans-splicing transduction, maybe because the neurons were already highly polarized when the tau isoform shift was induced (DIV11).

In summary, we provide novel mechanistic data about tau modulation of axonal transport in a relevant human neuronal setting that contributes to our understand of the highly regulated interplay of molecular motors in MT-dependent dynamics. We have shown that perturbations in the endogenous 3R:4R tau ratio are sufficient to impair the transport of the Alzheimer'sassociated molecule APP, suggesting a direct relationship between tau isoform imbalance and APP mislocalization that might underlie its pathological processing, leading to neuronal dysfunction. These data raise new perspectives about the modulation of abnormal tau metabolism as a potential therapeutic intervention for human tauopathies, including Alzheimer's disease.

\section{References}

Andreadis A (2005) Tau gene alternative splicing: expression patterns, regulation and modulation of function in normal brain and neurodegenerative diseases. Biochim Biophys Acta 1739:91-103. CrossRef Medline

Avale ME, Rodríguez-Martín T, Gallo JM (2013) Trans-splicing correction of tau isoform imbalance in a mouse model of tau mis-splicing. Hum Mol Genet 22:2603-2611. CrossRef Medline

Bodea LG, Eckert A, Ittner LM, Piguet O, Götz J (2016) Tau physiology and pathomechanisms in frontotemporal lobar degeneration. J Neurochem 138:71-94. CrossRef Medline

Bull ND, Guidi A, Goedert M, Martin KR, Spillantini MG (2012) Reduced axonal transport and increased excitotoxic retinal ganglion cell degeneration in mice transgenic for human mutant p301s tau. PLoS One 7:e34724. CrossRef Medline

Dawson HN, Cantillana V, Chen L, Vitek MP (2007) The tau N279K exon 10 splicing mutation recapitulates frontotemporal dementia and parkinsonism linked to chromosome 17 tauopathy in a mouse model. J Neurosci 27:9155-9168. CrossRef Medline

De Vos KJ, Grierson AJ, Ackerley S, Miller CC (2008) Role of axonal transport in neurodegenerative diseases. Annu Rev Neurosci 31:151-173. CrossRef Medline

Dixit R, Ross JL, Goldman YE, Holzbaur EL (2008) Differential regulation of dynein and kinesin motor proteins by tau. Science 319:1086-1089. CrossRef Medline

Ebneth A, Godemann R, Stamer K, Illenberger S, Trinczek B, Mandelkow E (1998) Overexpression of tau protein inhibits kinesin-dependent trafficking of vesicles, mitochondria, and endoplasmic reticulum: implications for Alzheimer's disease. J Cell Biol 143:777-794. CrossRef Medline

Encalada SE, Goldstein LS (2014) Biophysical challenges to axonal transport: motor-cargo deficiencies and neurodegeneration. Annu Rev Biophys 43:141-169. CrossRef Medline

Falzone TL, Stokin GB (2012) Imaging amyloid precursor protein in vivo: an axonal transport assay. Methods Mol Biol 846:295-303. CrossRef Medline

Falzone TL, Stokin GB, Lillo C, Rodrigues EM, Westerman EL, Williams DS, Goldstein LS (2009) Axonal stress kinase activation and tau misbehavior induced by kinesin-1 transport defects. J Neurosci 29:5758-5767. CrossRef Medline

Fu MM, Holzbaur EL (2013) JIP1 regulates the directionality of APP axonal transport by coordinating kinesin and dynein motors. J Cell Biol 202:495508. CrossRef Medline

Ghetti B, Oblak AL, Boeve BF, Johnson KA, Dickerson BC, Goedert M (2015) Invited review: Frontotemporal dementia caused by microtubule-associated protein tau gene (MAPT) mutations: a chameleon for neuropathology and neuroimaging. Neuropathol Appl Neurobiol 41:24-46. CrossRef Medline

Goedert M, Spillantini MG, Jakes R, Rutherford D, Crowther RA (1989) Multiple isoforms of human microtubule-associated protein tau: sequences and localization in neurofibrillary tangles of Alzheimer's disease. Neuron 3:519-526. CrossRef Medline

Goldsbury C, Thies E, Konzack S, Mandelkow EM (2007) Quantification of amyloid precursor protein and tau for the study of axonal traffic pathways. J Neurosci 27:3357-3363. CrossRef Medline

Goldstein LS (2012) Axonal transport and neurodegenerative disease: can we see the elephant? Prog Neurobiol 99:186-190. CrossRef Medline 
Goode BL, Chau M, Denis PE, Feinstein SC (2000) Structural and functional differences between 3-repeat and 4-repeat tau isoforms: implications for normal tau function and the onset of neurodegenerative disease. J Biol Chem 275:38182-38189. CrossRef Medline

Götz J, Ittner LM, Kins S (2006) Do axonal defects in tau and amyloid precursor protein transgenic animals model axonopathy in Alzheimer's disease? J Neurochem 98:993-1006. CrossRef Medline

Hendricks AG, Holzbaur EL, Goldman YE (2012) Force measurements on cargoes in living cells reveal collective dynamics of microtubule motors. Proc Natl Acad Sci U S A 109:18447-18452. CrossRef Medline

Hutton M, et al. (1998) Association of missense and 5' -splice-site mutations in tau with the inherited dementia FTDP-17. Nature 393:702-705. CrossRef Medline

Iovino M, Pfisterer U, Holton JL, Lashley T, Swingler RJ, Calo L, Treacy R, Revesz T, Parmar M, Goedert M, Muqit MM, Spillantini MG (2014) The novel MAPT mutation K298E: Mechanisms of mutant tau toxicity, brain pathology and tau expression in induced fibroblast-derived neurons. Acta Neuropathol 127:283-295. CrossRef Medline

Iovino M, Agathou S, González-Rueda A, Del Castillo Velasco-Herrera M, Borroni B, Alberici A, Lynch T, O’Dowd S, Geti I, Gaffney D, Vallier L, Paulsen O, Káradóttir RT, Spillantini MG (2015) Early maturation and distinct tau pathology in induced pluripotent stem cell-derived neurons from patients with MAPT mutations. Brain 138:3345-3359. CrossRef Medline

Ittner LM, Fath T, Ke YD, Bi M, van Eersel J, Li KM, Gunning P, Götz J (2008) Parkinsonism and impaired axonal transport in a mouse model of frontotemporal dementia. Proc Natl Acad Sci U S A 105:15997-16002. CrossRef Medline

Kanaan NM, Morfini G, Pigino G, LaPointe NE, Andreadis A, Song Y, Leitman E, Binder LI, Brady ST (2012) Phosphorylation in the amino terminus of tau prevents inhibition of anterograde axonal transport. Neurobiol Aging 33:826.e15-30. CrossRef Medline

Ke YD, Suchowerska AK, van der Hoven J, De Silva DM, Wu CW, van Eersel J, Ittner A, Ittner LM (2012) Lessons from Tau-deficient mice. Int J Alzheimers Dis 2012:873270. CrossRef Medline

Koo EH, Sisodia SS, Archer DR, Martin LJ, Weidemann A, Beyreuther K, Fischer P, Masters CL, Price DL (1990) Precursor of amyloid protein in Alzheimer disease undergoes fast anterograde axonal transport. Proc Natl Acad Sci U S A 87:1561-1565. CrossRef Medline

Leidel C, Longoria RA, Gutierrez FM, Shubeita GT (2012) Measuring molecular motor forces in vivo: implications for tug-of-war models of bidirectional transport. Biophys J 103:492-500. CrossRef Medline

Ma QL, Zuo X, Yang F, Ubeda OJ, Gant DJ, Alaverdyan M, Kiosea NC, Nazari S, Chen PP, Nothias F, Chan P, Teng E, Frautschy SA, Cole GM (2014) Loss of MAP function leads to hippocampal synapse loss and deficits in the Morris Water Maze with aging. J Neurosci 34:7124-7136. CrossRef Medline

Magnani E, Fan J, Gasparini L, Golding M, Williams M, Schiavo G, Goedert M, Amos LA, Spillantini MG (2007) Interaction of tau protein with the dynactin complex. EMBO J 26:4546-4554. CrossRef Medline

McVicker DP, Chrin LR, Berger CL (2011) The nucleotide-binding state of microtubules modulates kinesin processivity and the ability of Tau to inhibit kinesin-mediated transport. J Biol Chem 286:42873-42880. CrossRef Medline

McVicker DP, Hoeprich GJ, Thompson AR, Berger CL (2014) Tau interconverts between diffusive and stable populations on the microtubule surface in an isoform and lattice specific manner. Cytoskeleton 71:184194. CrossRef Medline

Mellone M, Kestoras D, Andrews MR, Dassie E, Crowther RA, Stokin GB, Tinsley J, Horne G, Goedert M, Tolkovsky AM, Spillantini MG (2013) Tau pathology is present in vivo and develops in vitro in sensory neurons from human P301S tau transgenic mice: a system for screening drugs against tauopathies. J Neurosci 33:18175-18189. CrossRef Medline

Mertens J, Stüber K, Poppe D, Doerr J, Ladewig J, Brüstle O, Koch P (2013) Embryonic stem cell-based modeling of tau pathology in human neurons. Am J Pathol 182:1769-1779. CrossRef Medline
Morris M, Maeda S, Vossel K, Mucke L (2011) The many faces of tau. Neuron 70:410-426. CrossRef Medline

Müller MJ, Klumpp S, Lipowsky R (2008) Tug-of-war as a cooperative mechanism for bidirectional cargo transport by molecular motors. Proc Natl Acad Sci U S A 105:4609-4614. CrossRef Medline

Otero MG, Alloatti M, Cromberg LE, Almenar-Queralt A, Encalada SE, Pozo Devoto VM, Bruno L, Goldstein LS, Falzone TL (2014) Fast axonal transport of the proteasome complex depends on membrane interaction and molecular motor function. J Cell Sci 127:1537-1549. CrossRef Medline

Panda D, Samuel JC, Massie M, Feinstein SC, Wilson L (2003) Differential regulation of microtubule dynamics by three- and four-repeat tau: implications for the onset of neurodegenerative disease. Proc Natl Acad Sci U S A 100:9548-9553. CrossRef Medline

Reis GF, Yang G, Szpankowski L, Weaver C, Shah SB, Robinson JT, Hays TS, Danuser G, Goldstein LS (2012) Molecular motor function in axonal transport in vivo probed by genetic and computational analysis in Drosophila. Mol Biol Cell 23:1700-1714. CrossRef Medline

Rodriguez-Martin T, Anthony K, Garcia-Blanco MA, Mansfield SG, Anderton BH, Gallo JM (2009) Correction of tau mis-splicing caused by FTDP-17 MAPT mutations by spliceosome-mediated RNA transsplicing. Hum Mol Genet 18:3266-3273. CrossRef Medline

Rodriguez-Martin T, Garcia-Blanco MA, Mansfield SG, Grover AC, Hutton M, Yu Q, Zhou J, Anderton BH, Gallo JM (2005) Reprogramming of tau alternative splicing by spliceosome-mediated RNA trans-splicing: implications for tauopathies. Proc Natl Acad Sci U S A 102:15659-15664. CrossRef Medline

Rodríguez-Martín T, Pooler AM, Lau DH, Mórotz GM, De Vos KJ, Gilley J, Coleman MP, Hanger DP (2016) Reduced number of axonal mitochondria and tau hypophosphorylation in mouse P301L tau knockin neurons. Neurobiol Dis 85:1-10. CrossRef Medline

Spillantini MG, Goedert M (2013) Tau pathology and neurodegeneration. Lancet Neurol 12:609-622. CrossRef Medline

Spillantini MG, Murrell JR, Goedert M, Farlow MR, Klug A, Ghetti B (1998) Mutation in the tau gene in familial multiple system tauopathy with presenile dementia. Proc Natl Acad Sci U S A 95:7737-7741. CrossRef Medline

Stamer K, Vogel R, Thies E, Mandelkow E, Mandelkow EM (2002) Tau blocks traffic of organelles, neurofilaments, and APP vesicles in neurons and enhances oxidative stress. J Cell Biol 156:1051-1063. CrossRef Medline

Stokin GB, Lillo C, Falzone TL, Brusch RG, Rockenstein E, Mount SL, Raman R, Davies P, Masliah E, Williams DS, Goldstein LS (2005) Axonopathy and transport deficits early in the pathogenesis of Alzheimer's disease. Science 307:1282-1288. CrossRef Medline

Tarhan MC, Orazov Y, Yokokawa R, Karsten SL, Fujita H (2013) Biosensing MAPs as "roadblocks": kinesin-based functional analysis of tau protein isoforms and mutants using suspended microtubules (sMTs). Lab Chip 13:3217-3224. CrossRef Medline

Terada S, Kinjo M, Aihara M, Takei Y, Hirokawa N (2010) Kinesin-1/ Hsc70-dependent mechanism of slow axonal transport and its relation to fast axonal transport. EMBO J 29:843-854. CrossRef Medline

Trinczek B, Ebneth A, Mandelkow EM, Mandelkow E (1999) Tau regulates the attachment/detachment but not the speed of motors in microtubuledependent transport of single vesicles and organelles. J Cell Sci 112:23552367. Medline

Vershinin M, Carter BC, Razafsky DS, King SJ, Gross SP (2007) Multiplemotor based transport and its regulation by Tau. Proc Natl Acad Sci U S A 104:87-92. CrossRef Medline

Yuan A, Kumar A, Peterhoff C, Duff K, Nixon RA (2008) Axonal transport rates in vivo are unaffected by tau deletion or overexpression in mice. J Neurosci 28:1682-1687. CrossRef Medline

Yuan A, Kumar A, Sasaki T, Duff K, Nixon RA (2013) Global axonal transport rates are unaltered in htau mice in vivo. J Alzheimers Dis 37:579586. CrossRef Medline

Zhang XQ, Zhang SC (2010) Differentiation of neural precursors and dopaminergic neurons from human embryonic stem cells. Methods Mol Biol 584:355-366. CrossRef Medline 